\title{
Magnetic quantum oscillations for the surface states of topological insulator $\mathrm{Bi}_{2} \mathrm{Se}_{3}$
}

\author{
Zhigang Wang, ${ }^{1}$ Zhen-Guo Fu, ${ }^{2,1}$ Shuang-Xi Wang, ${ }^{2,1}$ and Ping Zhang ${ }^{1,3, *}$ \\ ${ }^{1}$ LCP, Institute of Applied Physics and Computational Mathematics, \\ P.O. Box 8009, Beijing 100088, People's Republic of China \\ ${ }^{2}$ State Key Laboratory for Superlattices and Microstructures, \\ Institute of Semiconductors, Chinese Academy of Sciences, \\ P. O. Box 912, Beijing 100083, People's Republic of China \\ ${ }^{3}$ Center for Applied Physics and Technology, \\ Peking University, Beijing 100871, People's Republic of China
}

\begin{abstract}
We study quantum oscillations of the magnetization in $\mathrm{Bi}_{2} \mathrm{Se}_{3}(111)$ surface system in the presence of a perpendicular magnetic field. The combined spin-chiral Dirac cone and Landau quantization produce profound effects on the magnetization properties that are fundamentally different from those in the conventional semiconductor two-dimensional electron gas. In particular, we show that the oscillating center in the magnetization chooses to pick up positive or negative values depending on whether the zero-mode Landau level is occupied or empty. An intuitive analysis of these new features is given and the subsequent effects on the magnetic susceptibility and Hall conductance are also discussed.
\end{abstract}

PACS numbers: 73.20.At, 71.10.Ca, 72.15.Gd

\footnotetext{
${ }^{*}$ Corresponding author; zhang_ping@iapcm.ac.cn
} 
Magnetic oscillation, which was first predicted by Landau in 1930 [1], has been a focus in the condensed matter physics filed. One important reason is that the de Haas-van Alphen ( $\mathrm{dHvA}$ ) oscillations of magnetization provide a vigorous technique to study the properties of carriers around the Fermi surface. Especially in the last decade, thanks to the tremendous advances in microscopic semiconductor technology, the challenge encountered in the measurement of weak magnetization signal has been largely prominently overcome, and the magnetic dHvA oscillations in the two-dimensional electron gas (2DEG) systems have thus been extensively studied. For instance, Meinel et al. [2-4] developed dc superconducting quantum interference device magnetometers to study the dHvA oscillations in high-mobility semiconductor 2DEG. Schwarz et al. [5 7 7] studied the dHvA oscillations by using micromechanical cantilever magnetometers. Besides the purely orbital part, prominently, the influence [8] of the weak Rashba spin-orbit interaction (SOI) on the dHvA oscillations in the magnetization of the semiconductor 2DEG can also be effectively determined in experiment [9], which therefore opens a new door to measurement of the spintronic parameters in semiconductor heterostructures.

In the above-mentioned conventional semiconductor 2DEG systems, in which the electron motion is dominated by its orbital part, i.e., the magnetization oscillation mainly comes from the response of the electron $k$-quadratic kinetic energy to the external magnetic field. Although sometimes other factors than the pure kinetic energy, such as the spin-orbit interaction [8], have been taken into account, these factors in conventional semiconductor 2DEG systems play only a minor role. For example, they can result in a beating mode superposed onto the main dHvA oscillation pattern [9]. This situation, however, will be greatly changed by very recent theoretical finding [10] and experimental verification [11] of the topological insulators (TIs) with strong spin-orbit interaction. As a new state of matter as first addressed by Kane and Mele [12], the subject of time-reversal invariant TIs has attracted great attention in condensed-matter physics. Several three-dimensional (3D) solids, such as $\mathrm{Bi}_{1-x} \mathrm{Sb}_{x}$ alloys, $\mathrm{Bi}_{2} \mathrm{Se}_{3}$-family crystals, have been identified [13 17] to be strong TIs possessing anomalous band structures characterized by a $\mathbf{Z}_{2}$-valued topological invariant [12, 18]. This invariant, called $\nu_{0}$, counts the number of topologically protected gapless surface states (modulo 2). A non-zero invariant means that the surface of 3D TIs will be metallic. Instead of the conventional semiconductor 2DEG systems, the energy scale for the surface states of these 3D TIs is dominated by the $k$-linear spin-orbit interaction instead of the parabolic 

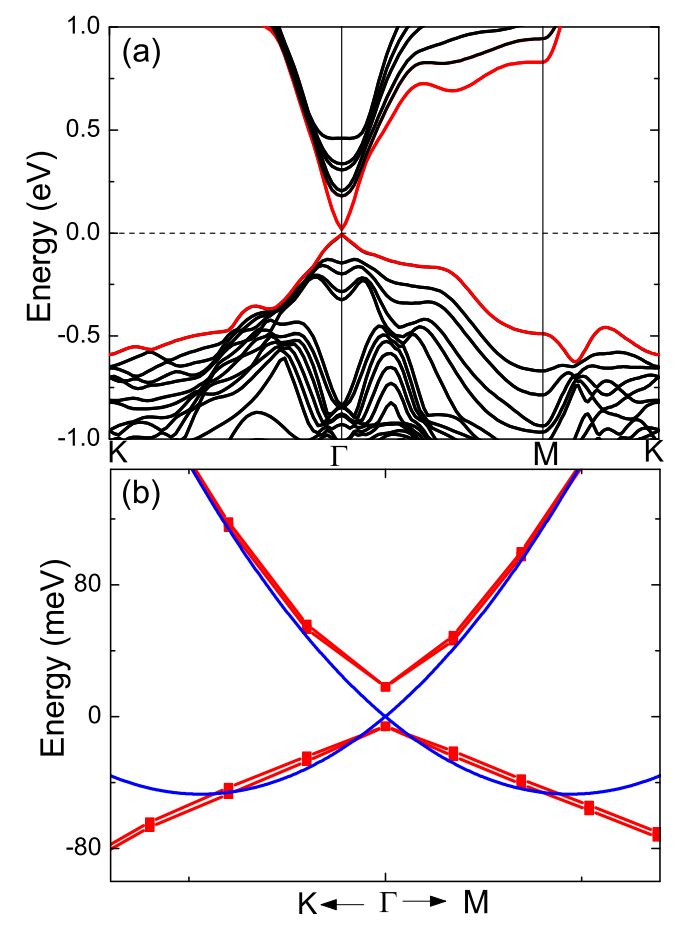

FIG. 1: (Color online) (a) The ab initio calculated band structure of the $\mathrm{Bi}_{2} \mathrm{Se}_{3}(111)$ thin film with the thickness of six quintuple layers. The red lines indicate the surface states while the black lines correspond to the bulk bands. (b) The fitting curves (blue lines) around the $\Gamma$ point with the model Hamiltonian [Eq. (1)].

kinetic energy. As a result, it is expected that the magnetic response properties of these topological surface states are fundamentally different from those of the conventional 2DEG.

Inspired by this observation, as well as by the recent experimental observation of the Landau quantization of the surface states of $\mathrm{Bi}_{2} \mathrm{Se}_{3}$ [19], in this paper we study the electron magnetic oscillations of these surface states. Specially, we consider the surface states of $\mathrm{Bi}_{2} \mathrm{Se}_{3}$. The first-principles surface band structure of $\mathrm{Bi}_{2} \mathrm{Se}_{3}$ is calculated by a simple supercell approach with spin-orbit coupling included and shown in Fig. 1(a) along the high-symmetry lines $(\boldsymbol{\Gamma} \rightarrow \mathbf{M}, \mathbf{M} \rightarrow \mathbf{K}$, and $\mathbf{K} \rightarrow \boldsymbol{\Gamma})$ in the surface Brillouin zone. In obtaining Fig. 11(a), here we have used Vienna ab-initio simulation package (VASP) [20]. The (Perdew-Burke-Ernzerhof) PBE [21] generalized gradient approximation and the projectoraugmented wave potential [22] were employed to describe the exchange-correlation energy and the electron-ion interaction, respectively. The SOI, which has been confirmed to play an important role in the electronic structure of $\mathrm{Bi}_{2} \mathrm{Se}_{3}$, was included during the calculation. 
The cutoff energy for the plane wave expansion was set to $300 \mathrm{eV}$. During the calculation, the experimental lattice constants [23] were adopted, i.e., $a=4.143 \AA, c=28.636 \AA$, with the internal parameter optimized automatically. The $\mathrm{Bi}_{2} \mathrm{Se}_{3}(111)$ surface was modeled by a slab composing of six quintuple layers (QLs) and a vacuum region of $20 \AA$. Integration over the Brillouin zone was done using the Monkhorst-Pack scheme [24] with $10 \times 10 \times 1$ grid points for surface calculations. The structures of bulk and slab were fully optimized until the maximum residual ionic force were below 0.01 and $0.02 \mathrm{eV} / \AA$, respectively. From Fig. 1(a) two chiral surface states are clearly seen to connect the conduction band and valence band, and cross each other to form a single Dirac-type contact at the $\Gamma$ point and aligning with the Fermi energy. The intrinsic defects such as the substitutional Bi defects at Se sites or the Se vacancies will play a role of $n$-doping, and consequently shift the Fermi level above the Dirac point. Note that due to the difference in the local symmetry between the top and bottom surfaces, there can develop an observable asymmetric charge distribution on the two surfaces if the sample is thin enough. This fact sometimes can open a small gap between the two spin chiral surface states as shown in Fig. 1(b) which presents an enlarged version of the surface bands around the Dirac cone. With increase of the thickness of the film, however, this asymmetry-induced Dirac gap tends to vanish, and this actually corresponds to the recent Landau quantization experiment, in which the used epitaxial $\mathrm{Bi}_{2} \mathrm{Se}_{3}$ is as thick as 50 QLs.

We report the calculated magnetization of the electrons on $\mathrm{Bi}_{2} \mathrm{Se}_{3}$ surface as a response to the external magnetic field. It is found that the magnetization oscillations in the present system differs from the traditional 2DEG by the fact that the dHvA oscillating center in $\mathrm{Bi}_{2} \mathrm{Se}_{3}$ departs from the well known (zero) value in the semiconductor 2DEG system. This departure has an intimate relation with the different Landau spectrum structures in these two kinds of systems. It is well known that the Landau levels (LL's) in the traditional 2DEG obey the $B(n+1 / 2)$ rule with $B$ being the external magnetic field and $n$ the LL index. However, the energy spectrum of the surface states in $\mathrm{Bi}_{2} \mathrm{Se}_{3}$ approximately obeys a $\sqrt{n B}$ rule. It is this difference in LLs that distributes differently in the two components of the magnetization, and eventually result in the different magnetic properties in these two systems. Furthermore, we have shown that the zero-mode LL plays a key role in determining the magnetization behavior in the TI surface systems.

The Hamiltonian describing the gapless surface states of $\mathrm{Bi}_{2} \mathrm{Se}_{3}$ can be approximately 
written as

$$
H(\mathbf{k})=\gamma k^{2}+\hbar v_{F}\left(k_{x} \sigma_{y}-k_{y} \sigma_{x}\right)
$$

where $v_{F}$ is the Fermi velocity and $\sigma$ are the Pauli matrices for surface-state electron spins. Note that this Hamiltonian has the same form as that of the conventional 2DEG system with Rashba spin-orbit coupling [8]. However, the intrinsic difference between these two kinds of systems is that the $k$-linear spin-orbit interaction is primary to the TI surface states, while the parabolic term is dominant in the conventional 2DEG. Although it is very simple, the Hamiltonian (2) is a general one, which can satisfactorily describe the gapless surface states of $\mathrm{Bi}_{2} \mathrm{Se}_{3}$ near the Dirac point. This satisfaction is particularly obvious for the upper part of the Dirac cone (electron part), as shown in Fig. 1(b), where Eq. (2) is used to fit the first-principles result, which gives $\gamma=2.1 \times 10^{2} \mathrm{meV} \mathrm{nm}^{2}$ and $\hbar v_{F}=200 \mathrm{meV} \mathrm{nm}$ (namely, $v_{F}=3.04 \times 10^{5} \mathrm{~m} / \mathrm{s}$ ). The effective mass $m^{*}$ is then obtained as $0.18 m_{e}$ according to $\gamma=\hbar^{2} / 2 m^{*}$, where $m_{e}$ is the mass of a free electron. The lower surface states (hole part) is not well described by Eq. (2) and a better fitting needs higher $k$-order corrections. For simplicity, and for the reason that we only concern the $n$-doping, here we neglect $\mathcal{O}\left(k^{3}\right)$ terms.

Let us now consider an external magnetic field $\mathbf{B}=B \hat{z}$ being perpendicular to the surface. Taking the Landau gauge for the vector potential, $A_{x}=B y$ and $A_{y}=0$, and the transform $\hbar \mathbf{k} \rightarrow \mathbf{\Pi}=\hbar \mathbf{k}+e \mathbf{A}$, one can obtain the following Hamiltonian

$$
H=\frac{\Pi^{2}}{2 m^{*}}+v_{F}\left(\Pi_{x} \sigma_{y}-\Pi_{y} \sigma_{x}\right)-\frac{1}{2} g_{s} \mu_{B} B \sigma_{z},
$$

where $g_{s}$ is the effective magnetic factor of the surface electron and $\mu_{B}$ is the Bohr magneton. For $\mathrm{Bi}_{2} \mathrm{Se}_{3}$-family (111) surfaces, the value of $g_{s}$ is approximately 8.0 [25]. Taking the fact that the system is translation invariant along the $x$ axis and therefore the wave number $k_{x}$ along this direction is a good quantum number, the Hamiltonian (2) can be rewritten as

$$
H=\hbar \omega_{c}\left(a^{\dagger} a+\frac{1-g \sigma_{z}}{2}+i \sqrt{2} \eta\left(a \sigma_{-}-a^{\dagger} \sigma_{+}\right)\right),
$$

where $\sigma_{ \pm}=\left(\sigma_{x} \pm i \sigma_{y}\right) / 2, \omega_{c}=e B / m^{*}, \eta=v_{F} m^{*} l_{B} / \hbar$ is the effective Rashba spin-orbit coupling with $l_{B}=\sqrt{\hbar / e B}$ being the magnetic length, $g=g_{s} m^{*} / 2 m_{e}$, and $a=\left[y+\left(\hbar k_{x}+i p_{y}\right) / e B\right] / \sqrt{2} l_{B}$ is the usual harmonic oscillator operator. The LLs are then given by

$$
E_{n}^{( \pm)}=\hbar \omega_{c}\left(n \pm \frac{1}{2} \sqrt{(1-g)^{2}+8 n \eta^{2}}\right)
$$


with $n=1,2, \cdots$. The $n=0$ LL only has the " + " branch, $E_{0}^{(+)}=\frac{|1-g|}{2} \hbar \omega_{c}$. The corresponding two-component eigenstates for $E_{n}^{( \pm)}$are given by

$$
|n\rangle^{( \pm)}=\left(\begin{array}{c}
\cos \theta_{n}^{( \pm)}|n\rangle \\
i \sin \theta_{n}^{( \pm)}|n-1\rangle
\end{array}\right)
$$

where $|n\rangle$ is the eigenstate of the $n$th LL of a free two-dimensional spinless electron. Here, $\tan \theta_{n}^{( \pm)}=-u_{n} \pm \sqrt{1+u_{n}^{2}}$ with $u_{n}=(1-g) / \sqrt{8 n} \eta$ when $n>0$ and $\theta_{0}^{(+)}=0$ for $n=0$. It is interesting to see that the $n=0$ LL has the fully polarized spin along the $z$ direction. Figure 2 plots the LLs as functions of the magnetic field. Note that although the LL equation (3) for $\mathrm{Bi}_{2} \mathrm{Se}_{3}$ surface states has a similar form with the conventional spin-orbit coupled semiconductor 2DEG [8], these two systems are fundamentally different by the amplitudes of the physical parameters. For the former the dimensionless parameter $\eta \gg 1$ while for the latter $\eta \ll 1$. Actually, for $\mathrm{Bi}_{2} \mathrm{Se}_{3}$, the Se-terminated (111) surface lattice constant is $a=4.143$ $\AA$. With this knowledge and through a normal fitting process, we obtain that at the external magnetic field $B=1 \mathrm{~T}, \hbar \omega_{c}=0.61 \mathrm{meV}$ and $\eta=12.4$. However, for a conventional 2DEG system with Rashba coupling, the dimensionless parameter $\eta$ is typically in the range $0 \sim 0.2$. Based on this fact, the energy spectrum (3) can be well approximated by the dispersion relation

$$
\begin{aligned}
& E_{n}^{( \pm)}= \pm \hbar \omega_{c} \sqrt{2 n \eta^{2}+\frac{g^{2}}{4}}= \pm v_{F} \sqrt{2 n e \hbar B+\delta^{2}} \quad(n \neq 0), \\
& E_{0}^{(+)}=\operatorname{sgn}\left(g_{s}\right) v_{F}|\delta|
\end{aligned}
$$

where $\delta=g_{s} \mu_{B} B / 2 v_{F}$. Because the Zeeman splitting is much smaller than the LL separations (for example, $g=0.72$ when $g_{s}$ takes the value 8 , resulting in $\frac{1}{2} g_{s} \mu_{B} B=0.13 \mathrm{meV}$ at $\mathrm{B}=1 \mathrm{~T}$ ), thus the effect of the Zeeman term on the $n \neq 0$ LLs is very tiny and can be safely neglected in considering the electron occupation of $n \neq 0$ LLs. It is not so, however, for the $n=0$ LL. In fact, in the absence of the Zeeman splitting, the Dirac-Landau energy spectrum (5) is massless with a whole electron-hole symmetry and only half of the zero mode is occupied by electrons in the case of $n$-doping. If the Zeeman splitting is finite, the spectrum (5) is massive and the $n=0$ LL shifts upward or downward, depending on the orientation of the exchange field (the sign of $g_{s}$ ). Correspondingly, this "zero" mode will be saturated by electrons for $g_{s}>0$ or empty for $g_{s}<0$, which, as shown in the following discussion, will greatly influence the behavior of the magnetic response of the system. 


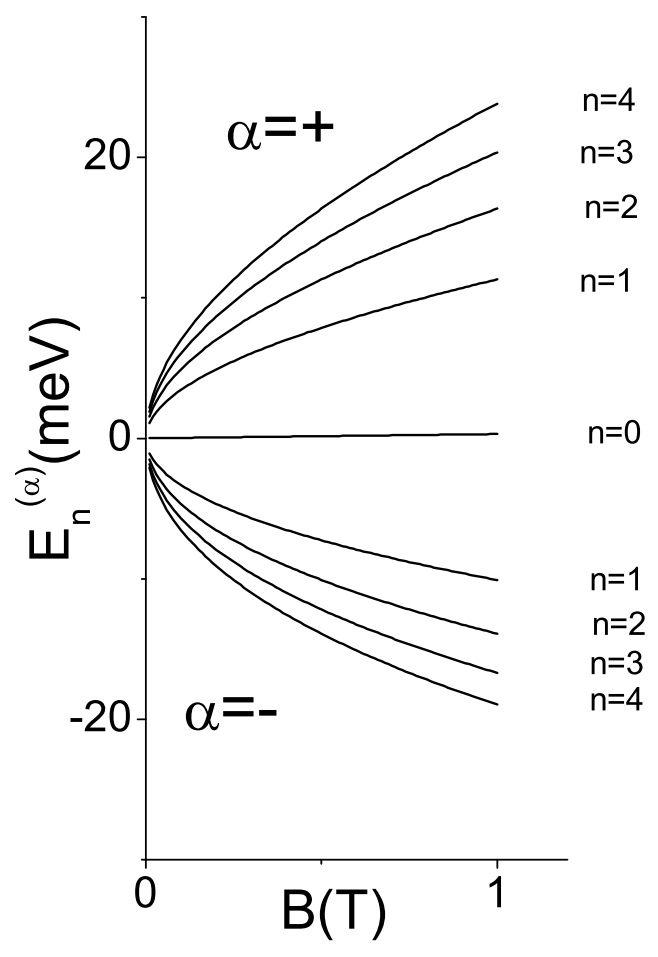

FIG. 2: The Landau levels in the $\mathrm{Bi}_{2} \mathrm{Se}_{3}(111)$ surface system as functions of the external magnetic field $B$.

Now with the LL spectrum (5) [or the $k^{2}$-corrected LL spectrum (3)], we study the magnetization of the surface electrons of $\mathrm{Bi}_{2} \mathrm{Se}_{3}$. The magnetization density is the derivative of the Helmholtz free energy density with respect to $B$ at fixed electron density $\mathcal{N}$ and temperature $T, M=-\left.(\partial F / \partial B)\right|_{\mathcal{N}, T}$. The Helmholtz free energy density is given by

$$
\begin{aligned}
F(B, T) & =\mu \mathcal{N}-\frac{N_{\nu}}{\beta} \sum_{n=1}^{\infty} \ln \left[1+e^{\beta\left(\mu-E_{n}^{(+)}\right)}\right] \\
& -\frac{N_{0}}{\beta} \ln \left[1+e^{\beta\left(\mu-E_{0}^{(+)}\right)}\right],
\end{aligned}
$$

where $\beta=1 / k_{B} T, N_{\nu}=1 / 2 \pi l_{B}^{2}$ is the degeneracy for a non-zero LL (namely, the number of electrons per unit area on a LL), and $\mu$ is the electron chemical potential. The second line in Eq. (6) denotes the contribution from the $n=0 \mathrm{LL}$ and there exist three possibilities for its contribution: (i) If this level is exactly a zero mode, $E_{0}^{(+)}=0$, then the system has the electron-hole symmetry and half of the particles in the zero mode are electrons. In this case $N_{0}=N_{\nu} / 2$; (ii) If the Zeeman splitting cannot be neglected and the $g$ factor is positive as Eq. (3) shows, then the $n=0$ LL shifts upward and is fully accessible to electrons. In this case 
$N_{0}=N_{\nu}$; (iii) Otherwise, if the $g$ factor is negative, then the $n=0 \mathrm{LL}$ shifts downward and is unavailable to electron occupation. In this case $N_{0}=0$. The $B$-dependent chemical potential $\mu$ in Eq. (6) is connected to the experimentally accessible electron density $\mathcal{N}$, which is given by

$$
\mathcal{N}=N_{\nu} \sum_{n=1}^{\infty} f_{n}^{(+)}+N_{0} f_{0}^{(+)}
$$

with $f_{n}^{(+)}=1 /\left[e^{\beta\left(E_{n}^{(+)}-\mu\right)}+1\right]$ being the Fermi-Dirac distribution of the LL $E_{n}^{(+)}$. From Eq. (6) the electron magnetization density becomes

$$
\begin{aligned}
M & =-\left(\sum_{n=1}^{\infty} N_{\nu} f_{n}^{(+)} \frac{\partial E_{n}^{(+)}}{\partial B}+N_{0} f_{0}^{(+)} \frac{\partial E_{0}^{(+)}}{\partial B}\right) \\
& +\left(\frac{e}{h} \sum_{n=1}^{\infty} \frac{1}{\beta} \ln \left[1+e^{\beta\left(\mu-E_{n}^{(+)}\right)}\right]+\frac{1}{\beta} \frac{\partial N_{0}}{\partial B} \ln \left[1+e^{\beta\left(\mu-E_{0}^{(+)}\right)}\right]\right) \\
& \equiv M^{(0)}+M^{(1)} .
\end{aligned}
$$

The first part $M^{(0)}$ is the conventional contribution from the $B$ dependence of the LLs and thus denotes a diamagnetic response. The second part $M^{(1)}$ comes from the $B$ dependence of the level degeneracy factor $N_{\nu}$, thus describing the effect of the variation in the density of states upon the magnetic field and denoting a paramagnetic contribution to the total magnetization. Obviously, $M^{(0)}$ is negative while $M^{(1)}$ is positive, the net result is an oscillation of the total magnetization $M$ as a function of $B$.

We plot in Figs. 3(a) and 3(b) the magnetic dHvA oscillations of the chemical potential and magnetization in $\mathrm{Bi}_{2} \mathrm{Se}_{3}$ for the above-mentioned three cases of zero-mode filling. Comparing to the well-known dHvA oscillating pattern in the conventional 2DEG, one can immediately obtain three prominent features in the present TI surface system: (i) Although the oscillating center of the chemical potential $\mu$ keeps a fixed value unchanged by changing the external magnetic field $B$ when the $n=0$ LL is exactly a zero mode, it linearly increases/decreases with $B$ when the $g$ factor is positve/negative; (ii) The oscillating center of the magnetization $M$ keeps a non-zero value unchanged by varying the external magnetic field strength. This is totally different from those in the semiconductor 2DEG. It is well known that in the clean semiconductor 2DEG sample, the oscillating center of the chemical potential $\mu$ keeps a constant value unchanged and that of the magnetization $M$ keeps zero unchanged when varying the magnetic field $B$; (iii) The magnetization for the case of 

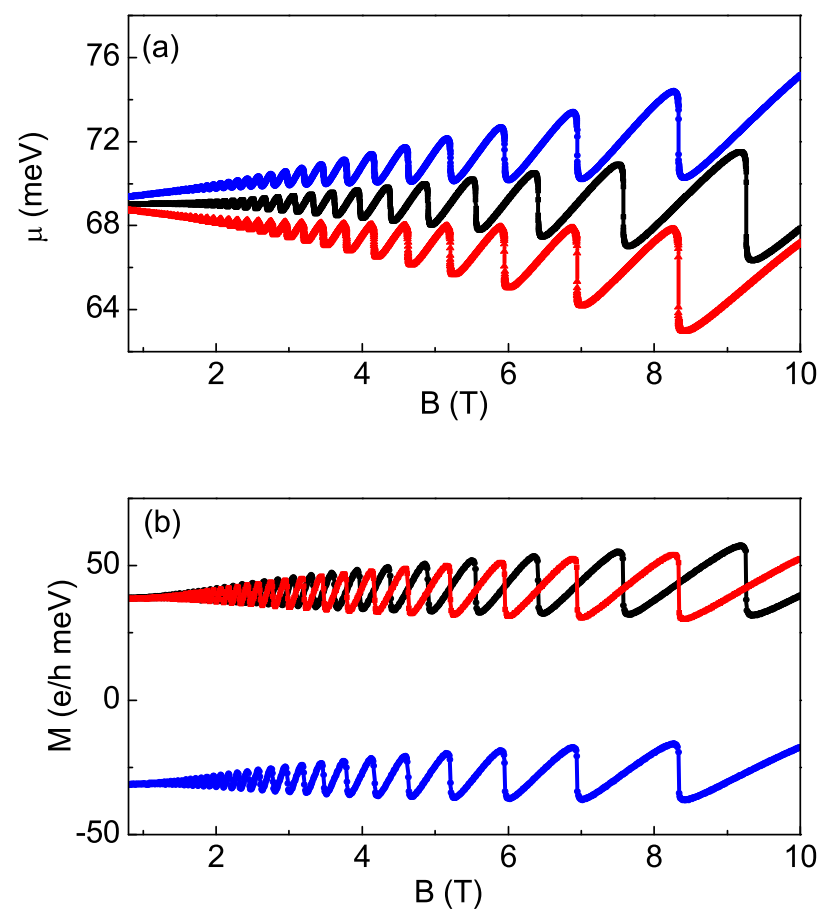

FIG. 3: (Color online) Magnetic field dependence of (a) chemical potential $\mu$ and (b) magnetization $M$ in $n$-doped $\mathrm{Bi}_{2} \mathrm{Se}_{3}(111)$ surface system with electron density $\mathcal{N}=1.0 \times 10^{16} \mathrm{~m}^{-2}$. The temperature is set as $k_{B} T=0.3 \mathrm{meV}$. The black, red, and blue curves correspond to the cases that the zero mode is half filled, saturated, and empty, respectively.

empty zero mode is fundamentally distinguished from the cases of saturated and half-filling zero mode by a total sign inversion. In addition, the magnetic oscillation patterns for the cases of saturated and half-filling zero mode are out phase. Thus, Fig. 3 clearly reveals the fundamental role the zero mode played in determining the magnetic response properties of the TI surface states.

For further illustration and to see the origin of the sign inversion in the magnetization when the zero is changed from filling to unfilling, let us first consider the case of saturated zero mode. In this case the $n=0 \mathrm{LL}$ is occupied by electrons with the degeneracy $N_{0}=N_{\nu}$. The discrepancy in the dHvA oscillating patterns between the TI surface and the semiconductor 2DEG comes from their different energy dispersion relations. The former versus the external magnetic field obeys square root rule while the latter obeys linear rule. It is well known that at zero temperature, the two components of the magnetization in the conventional 2DEG turn to be $M^{(0)}=-\frac{e}{h} \sum_{n=0}^{\text {occu. }} E_{n}$ and $M^{(1)}=\frac{e}{h} \sum_{n=0}^{\text {occu. }}\left(\mu_{0}-E_{n}\right)$, respectively. 
Here $\mu_{0}$ is the zero-temperature Fermi energy and the LL $E_{n}^{(+)} \propto B$. The negative $M^{(0)}$ and the positive $M^{(1)}$ gives that the net result is an oscillation of the total magnetization $M$ as a function of $B$. The oscillation amplitude increases with $B$ and the oscillation center is zero. However, because the LL $E_{n} \propto \sqrt{B}$ for the present system, at zero temperature the first component of $M$ turns to be $M^{(0)}=-\frac{e}{h} \sum_{n=0}^{\text {occu. }} \frac{E_{n}^{(+)}}{2}$, while the second component is $M^{(1)}=\frac{e}{h} \sum_{n=0}^{\text {occu. }}\left(\mu_{0}-E_{n}^{(+)}\right)$. By comparison with those in the semiconductor 2DEG, one can find that in the present TI surface system the diamagnetic contribution $\left(M^{(0)}\right)$ is reduced. As a result, the oscillating center of the magnetization is now a positive value for the case of saturated zero mode. This simple comparison is not strict in mathematics, however, it affords an intuitive explanation on the difference of the magnetization between the TI surface and the semiconductor 2DEG.

In the case of empty zero mode, the $n=0 \mathrm{LL}$ is excluded and the first component $M^{(0)}$ now becomes $M^{(0)}=-\frac{e}{h} \sum_{n=1}^{\text {occu. }} \frac{E_{n}^{(+)}}{2}$, while the second component becomes $M^{(1)}=\frac{e}{h} \sum_{n=1}^{\text {occu. }}\left(\mu_{0}-\right.$ $\left.E_{n}^{(+)}\right)$. Compared to the case of saturated zero mode, and considering $\mu_{0} \gg E_{0}^{(+)}$, one can find that the magnetization in the case of the empty zero mode is smaller than the saturated case by a quantity $\frac{e}{h} \mu_{0}$ and therefore becomes negative during its oscillations as a function of $B$.

Note that the abrupt jump in the dHvA oscillation is on the high magnetic field side of the sawtooth, which is special for our present choice of the thermodynamic system. If the system is constrained to have constant chemical potential, then the jump in the dHvA oscillation will move to the low magnetic field side of the sawtooth, which has been confirmed by Meinel et al. [2] in an experiment with the electron density $\mathcal{N}$ modulated by applying a gate voltage to the sample.

The above discussions on the dHvA oscillations of the magnetization focus on the situation that the total number of electrons on the LL's is field independent. Now we consider the magnetization properties in another situation that the chemical potential is field independent. Figure 4(a) plots the magnetization as a function of the zero-temperature chemical potential (i.e., the Fermi energy) at $B=4 \mathrm{~T}$ for three cases of zero-mode filling. The dHvA oscillating patterns as a function of the chemical potential can be observed from Fig. 4(a). There exist different kinds of patterns for the dHvA oscillating center in different cases. When the $n=0 \mathrm{LL}$ is half occupied by electrons, the dHvA oscillating center keeps zero unchanged by increasing the chemical potential. When the zero mode is saturated/empty, 

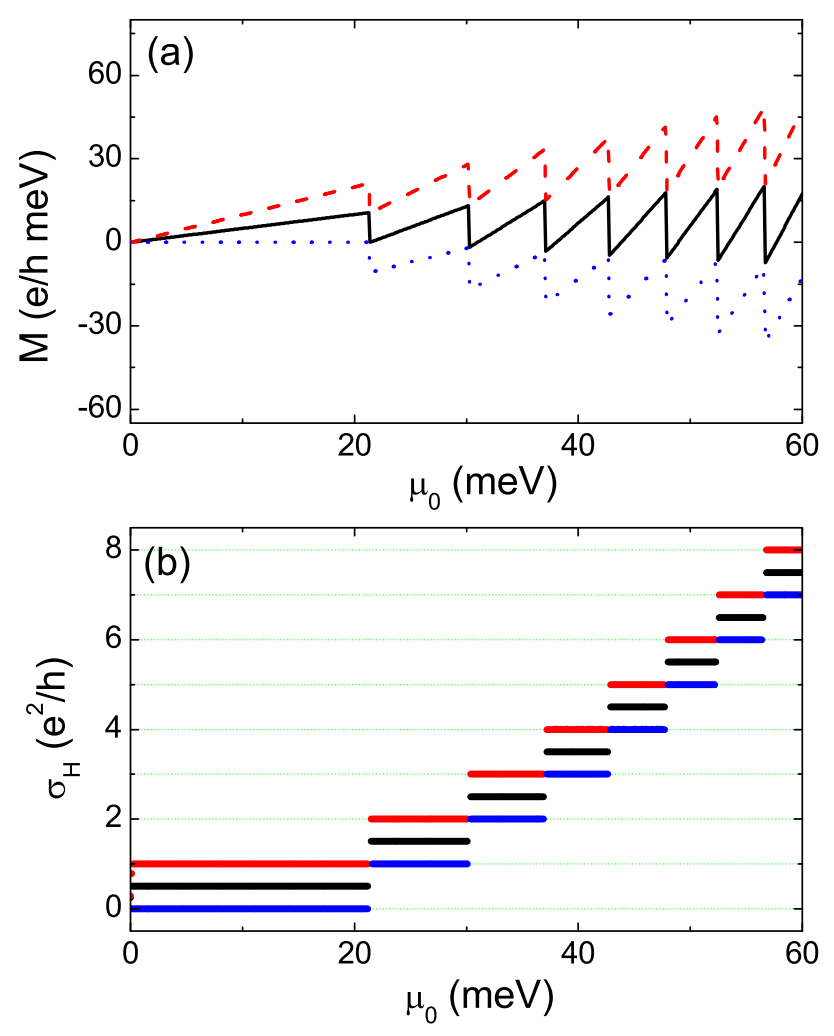

FIG. 4: (Color online) (a) Calculated magnetization as a function of the Fermi energy $\mu_{0}$. The external magnetic field is chosen as $B=4 \mathrm{~T}$. (b) The derived Hall conductance from $\partial M / \partial \mu_{0}$. The black, red, and blue curves correspond to the cases that the zero mode is half filled, saturated, and empty, respectively.

the dHvA oscillating center linearly increases/decreases by increasing the chemical potential. A fact that should be addressed is that because the chemical potential is tuned freely and independent with the external field, there are no phase difference in the dHvA oscillations for different cases. The corresponding $\partial M / \partial \mu_{0}$ are also calculated, from which the Hall conductance $\sigma_{H}$ is obtained by combining the thermodynamic relationship and Streda formula:

$$
\partial M / \partial \mu_{0}=\frac{1}{e} \sigma_{H}
$$

The result of Hall conductance as a function of the Fermi energy is plotted in Fig. 4(b), from which Hall plateaus can be clearly seen. The plateau values of $\sigma_{H}$ depend on the zero-mode filling. If the $n=0 \mathrm{LL}$ is half filled, the Hall conductance takes half-integer values of $\sigma_{H}=(n+1 / 2) e^{2} / h$, as shown in Fig. 4(b) by black step lines. To date, measuring the 
half-integer quantum Hall effect on the TI surfaces keeps a challenging task, although the LLs have been recently observed [19]. If the zero mode is saturated, then $\sigma_{H}=(n+1) e^{2} / h$, as shown in Fig. 4(b) by red step lines. Finally, if the zero mode is empty, then $\sigma_{H}=(n-1) e^{2} / h$, as shown in Fig. 4(b) by blue step lines. We note that the quantum Hall effect in the TI surface system with finite sample size has also been discussed in Ref. [26].

The information on the magnetic susceptibility $\chi$ of the TI surfaces, which is defined as the derivative of the magnetization with respect to the external magnetic field, $\chi=\partial M / \partial B$, can be easily obtained with the knowledge of the magnetization [Eq. (8)]. The final expression of the magnetic susceptibility is given by

$$
\begin{aligned}
\chi & =\left[\frac{e}{h} \sum_{n=1}^{\infty} f_{n}^{(+)}\left(\frac{\partial \mu}{\partial B}-2 \frac{\partial E_{n}^{(+)}}{\partial B}\right)+\frac{\partial N_{0}}{\partial B} f_{0}^{(+)}\left(\frac{\partial \mu}{\partial B}-2 \frac{\partial E_{0}^{(+)}}{\partial B}\right)\right] \\
& -\left\{\sum_{n=1}^{\infty} N_{\nu}\left[\frac{\partial f_{n}^{(+)}}{\partial B} \frac{\partial E_{n}^{(+)}}{\partial B}+f_{n}^{(+)} \frac{\partial^{2} E_{n}^{(+)}}{\partial B^{2}}\right]+N_{0}\left[\frac{\partial f_{0}^{(+)}}{\partial B} \frac{\partial E_{0}^{(+)}}{\partial B}+f_{0}^{(+)} \frac{\partial^{2} E_{0}^{(+)}}{\partial B^{2}}\right]\right\} .
\end{aligned}
$$

Figure 5 plots the magnetic susceptibility in $\mathrm{Bi}_{2} \mathrm{Se}_{3}$ sample as a function of the inverse magnetic field, $1 / B$. Because the resonant (for magnetic susceptibility) magnetic field only reflects the occupation of the LLs, which is the same as that in the conventional 2DEG, it losses the message on the oscillating center value of the magnetization. However, the difference between the half-filled case and saturated/empty case of the zero mode is still clearly revealed in this figure.

In summary, we have investigated the dHvA oscillations of the magnetization in the $\mathrm{Bi}_{2} \mathrm{Se}_{3}$-family surface systems. Our results show that the dHvA oscillating center of the magnetization maintains positive values when the $n=0 \mathrm{LL}$ is fully occupied or half occupied. When this mode is empty, the dHvA oscillating center changes a sign. These results are fundamentally different from those in the conventional semiconductor 2DEG systems, in which the dHvA oscillating center is at zero. We have given an intuitive analysis on this difference, which turns to have an intimate relation with different forms of the energy dispersions in these two kinds of systems. This can be reflected by the fact that the LLs for the TI surfaces is proportional to $\sqrt{B}$ instead of $B$-linear dependence accommodated by the conventional 2DEG. Furthermore, the essential role that the zero mode played has been illustrated by the different behavior of the Hall conductance at three kinds of electron occupation of this mode. We expect that the present results for the topologically nontrivial features in the magnetic response of the TI surfaces can be experimentally confirmed in the 


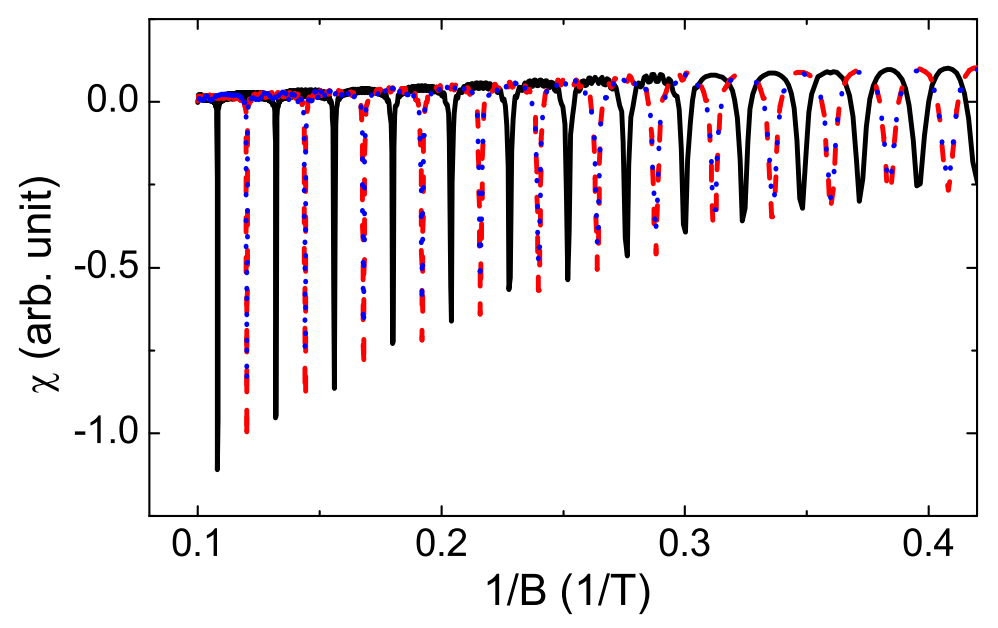

FIG. 5: (Color online) Calculated magnetic susceptibility $\chi$ as a function of $1 / B$. The parameters are the same as those in Fig. 3. The black, red, and blue curves correspond to the cases that the zero mode is half filled, saturated, and empty, respectively.

future topological magnetoelectric studies.

Note added.- While this work was completed, we were aware of an experimental measurement [27] of the magnetization for the topological insulator $\mathrm{Bi}_{1-x} \mathrm{Sb}_{x}(0.07<x<0.22)$. Compared to $\mathrm{Bi}_{2} \mathrm{Se}_{3}$, the surface band structure of $\mathrm{Bi}_{1-x} \mathrm{Sb}_{x}$ alloy is much more complicated. Furthermore, in $\mathrm{Bi}_{1-x} \mathrm{Sb}_{x}$ the bulk band is often coupled with surface band during measurement. These facts make it difficult to study the magnetic quantum oscillations that are fully from the 2D surface states of $\mathrm{Bi}_{1-x} \mathrm{Sb}_{x}$ alloy. In spite of these complicated facts, we expect that the phenomenon of large-amplitude dHvA magnetic oscillations found in Ref. [27] is closely related to our theoretical finding in the present paper.

\section{Acknowledgments}

This work was supported by NSFC under Grants No. 90921003, No. 10904005 and No. 60776063, and by the National Basic Research Program of China (973 Program) under Grant No. 2009CB929103.

[1] L.D. Landau, Z. Phys. 64, 629 (1930). 
[2] I. Meinel, T. Hengstmann, D. Grundler, D. Heitmann, W. Wegscheider, and M. Bichler, Phys. Rev. Lett. 82, 819 (1999).

[3] I. Meinel, D. Grundler, D. Heitmann, A. Manolescu, V. Gudmundsson, W. Wegscheider, and M. Bichler, Phys. Rev. B 64, 121306(R) (2001).

[4] I. Meinel, D. Grundler, S. Bargstadt-Franke, Ch. Heyn, and D. Heitmann, Appl. Phys. Lett. 70, 3305 (1997).

[5] M. A. Wilde, M. P. Schwarz, Ch. Heyn, D. Heitmann, D. Grundler, D. Reuter, and A. D. Wieck, Phys. Rev. B 73, 125325 (2006).

[6] M. P. Schwarz, D. Grundler, I. Meinel, Ch. Heyn, and D. Heitmann, Appl. Phys. Lett. 76, 3564 (2000).

[7] M. P. Schwarz, M. A. Wilde, S. Groth, D. Grundler, Ch. Heyn, and D. Heitmann, Phys. Rev. B 65, 245315 (2002).

[8] Z. Wang, W. Zhang, and P. Zhang, Phys. Rev. B 79, 235327 (2009).

[9] M. A. Wilde, D. Reuter, Ch. Heyn, A. D. Wieck, and D. Grundler, Phys. Rev. B 79, 125330 (2009).

[10] B. A. Bernevig, T. L. Hughes, and S. C. Zhang, Science 314, 1757 (2006).

[11] M. König, S. Wiedmann, Christoph Brüne, A. Roth, H. Buhmann, L. W. Molenkamp, X.-L. Qi, and S. C. Zhang, Science 318, 766 (2007).

[12] C. L. Kane, and E. J. Mele, Phys. Rev. Lett. 95, 146802 (2005).

[13] L. Fu and C. L. Kane, Phys. Rev. B 76, 045302 (2007).

[14] D. Hsieh, D. Qian, L. Wray, Y. Xia, Y. S. Hor, R. J. Cava, and M. Z. Hasan, Nature 452, $970(2008)$.

[15] H. J. Zhang, C. X. Liu, X. L. Qi, X. Dai, Z. Fang, and S. C. Zhang, Nature Phys. 5, 438 (2009).

[16] Y. Xia, D. Qian, D. Hsieh, L. Wrayl, A. Pal1, H. Lin, A. Bansil, D. Grauer, Y. S. Hor, R. J. Cava, Nat. Phys. 5, 398 (2009).

[17] Y. L. Chen, J. G. Analytis, J. H. Chu, Z. K. Liu, S. K. Mo, X. L. Qi, H. J. Zhang, D. H. Lu, X. Dai, Z. Fang, Science 325, 178 (2009).

[18] L. Fu, C. L. Kane and E. J. Mele, Phys. Rev. Lett. 98, 106803 (2007).

[19] P. Cheng, C. Song, T. Zhang, Y. Zhang, Y. Wang, J.-F. Jia, J. Wang, Y. Wang, B.-F. Zhu, X. Chen, X. Ma, K. He, L. Wang, X. Dai, Z. Fang, X. C. Xie, X. L. Q, C. X. Liu, S. C. Zhang, 
and Q. K. Xue, arXiv:1001.3220.

[20] G. Kresse and J. Furthmuller, Phys. Rev. B 54, 11169 (1996).

[21] J. P. Perdew, K.Burke, and M. Ernzerhof, Phys. Rev. Lett. 77, 3865 (1996).

[22] G. Kresse and D. Joubert, Phys. Rev. B 59, 1758 (1999).

[23] S. Nakajima, J. Phys. Chem. Solids 24, 479 (1963).

[24] H. J. Monkhorst and J. D. Pack, Phys. Rev. B 13, 5188 (1976).

[25] C.-X. Liu, X.-L. Qi, H. Zhang, X. Dai, Z. Fang, and S.-C. Zhang, arXiv:1005.1682,

[26] S.-Q. Shen, arXiv:0909.4125.

[27] A. A. Taskin and Y. Ando, Phys. Rev. B 80, 085303 (2009). 